\begin{tabular}{|c|c|c|}
\hline ITC $1 / 49$ & \multicolumn{2}{|c|}{ Applications of Optimal Control to Production Planning } \\
\hline $\begin{array}{l}\text { Information Technology } \\
\text { and Control }\end{array}$ & Received 2019/03/01 & Accepted after revision 2019/11/19 \\
\hline $\begin{array}{l}\text { Vol. } 49 \text { / No. } 1 \text { / } 2020 \\
\text { pp. } 89-99 \\
\text { DOI /10.5755/j01.itc.49.1.23891 }\end{array}$ & \multicolumn{2}{|c|}{ Cross $\mathrm{http} / / / \mathrm{dx}$. doi.org//10.5755/j01.itc.49.1.23891 } \\
\hline
\end{tabular}

HOW TO CITE: Popescu, L. (2020). Applications of Optimal Control to Production Planning. Information Technology and Control, 49(1), 89-99. https://doi.org//10.5755/j01.itc.49.1.23891

\title{
Applications of Optimal Control to Production Planning
}

\section{Liviu Popescu}

Department of Statistics and Economic Informatics, Faculty of Economics and Business Administration, University of Craiova; Al. I. Cuza Str. 13, 200585, Craiova, Romania; phone: +0724 3546 35; e-mails:liviupopescu@central.ucv.ro,liviunew@yahoo.com

Corresponding author: liviunew@yahoo.com

In this paper a problem of optimization and production planning using the optimal control methods and Pontryagin Maximum Principle is solved. An economic model is proposed and an optimal plan of production for $n$ products is found, to ensure the required quantity at specified delivery data with minimum cost of inventory and production. It is proved that the economic system is not controllable, in the sense that it is not possible to reach any final stock quantity. Finally, this construction is justified with two numerical examples.

KEYWORDS: optimal control, controllability, Pontryagin Maximum Principle, Hamilton-Jacobi-Bellman equations, production planning, inventory and production.

\section{Introduction}

Control theory is frequently used in modelling and analysis of operational systems in production planning and logistics. Moreover, there can be found numerous applications to supply chain engineering and management. In the last decades, Lie methods have been applied successfully in the study of controllability of optimal control problems. Brocket [6] has proved the connection between the Lie theory and optimal control. In his book, Sontag [27] studied nonlinear controllability via Lie-algebraic methods. Agrachev and Sachkov [1] presented some methods of the mathematical control theory treated from the geometric point of view. LaValle [17] gave an unified treatment of control theory including control affine systems and Popescu [22] used the framework of Lie algebroids in the study of driftless control affine systems. One of the motivations for this work is the study of Lagrangian systems with some external constraints. These systems have a wide application in many different areas as optimal control theory, econometrics, cybernetics 
or operational research (see, for instance, Arrow [2], Seierstad and Sydsater [23], Sethi and Thompson [26], Caputo [7], Weber [30], Hermosilla,Vinter and Zidani [12], Varjani, Shamsi and Malek [29], Milosz et al. [19]). The present paper is organized as follows. In the second section, an overview of earlier research concerning optimal control theory applications in production economics is given. In Section 3, the known results about optimal control theory for control affine systems are presented, including the controllability problems in the driftless case and involutive distributions. In Section 4, an application of optimal control to a problem of inventory and production planning is given. In Section 5, two numerical examples are presented. The Pontryagin Maximum Principle will be used in order to find the optimal solution. The case of one single product studied by Kamien and Schwartz in [15] is extended. Moreover, it is shown that the distribution generated by the vector fields is involutive and it determines a foliation in the state space. It is proved that the economic system is not controllable, in the sense that it is not possible to reach any final stock quantity. In the last part of the paper the complete solution of the problem is found, using a convenient change of variables for the system of differential equations generated by Pontryagin Maximum Principle. Finally, two numerical examples in the particular cases $n=3$ and $n=4$ are presented.

\section{Optimal Control and Pontryagin Maximum Principle}

A large class of control theory applications to business and economics can be explained by a rigorous quantitative foundation including stability of controlled processes and non-linear systems, controllability and observability (Pontryagin et al. [21], Lee and Markus [18], Bellman [4], Sethi [25], Sethi and Thompson [26]). Optimal control problems belong to the class of extremum optimization theory, i.e., minimization or maximization of some functions equipped with some external constraints. This theory extends classical calculus variation theory that is based on control variations of a continuous trajectory developed by Euler, Lagrange, Hamilton, Jacobi et al. In the 20th century, two computational fundamentals of optimal control theory, the maximum principle (Pontryagin et al., [21]) and the dynamic programming method (Bellmann [4]) were developed. One of the most important methods in the analysis of solutions for the optimal control problems is provided by Pontryagin's Maximum Principle. A curve $c(t)=(x(t), u(t))$ is an optimal trajectory if there exists a lifting of $x(t)$ to the dual space $(x(t), p(t))$ satisfying the Hamilton-Jacobi-Bellman equations. However, finding a complete solution to an optimal control problem remains extremely difficult for several reasons. Firstly, the problem of integrating a Hamiltonian system, which is generally difficult to integrate, except for particular dynamics and costs is approached. Secondly, even though all solutions are found, there remains the problem of selecting optimal solutions from them. The article by Hwang, Fan and Erikson [13] determined the use of optimal control action for production planning and the corresponding trajectory of state variables by means of the Maximum Principle subject to the minimization of costs. In this paper, the solution of a problem of optimization and production planning using the optimal control techniques and Pontryagin's Maximum Principle is found.

A mathematical model of an economic problem is proposed in order to find an optimal plan of production for $n$ products and to ensure the required quantity at specified delivery data at minimum cost of inventory and production. Some percentages from the quantities of $n-1$ products are used in the manufacture of the last product. Such type of production problems with different constraints are intensely studied. Thus, Sethi [25] applied the Maximum Principle to some problems of production and inventory. Axsäter [3] gave an overview of earlier research concerning control theory applications in production and inventory control. Feichtinger and Hartl [9] studied the problem of simultaneously determining the optimal price policy and production rate over a given planning horizon. Gaimon [10] considered a profit maximizing firm that derives the optimal price for its output, level of output, level of inventory and composition of productive capacity over time. Kogan and Khmelnitsky [16] presented an optimal control model for continuous time production and setup scheduling. Ortega and Lin [20] presented a review of control theory applications to the production-inventory problem. Benjaafar, Gayon and Tepe [5] considered the control of a production-inventory system with impatient customers. Dolgui et al. [8] presented some of the existing literature of supply planning tools 
under uncertainty of lead times. Schwartz and Rivera [28] gave a practical approach for applying control-theoretic principles to tactical inventory management problem in a production-inventory system, the basic unit in a supply chain. Janušauskaitè [14] investigated two mathematical models of multistage inventory control processes with continuous and discrete density functions of demands. Gayon, Vercraene and Flapper [11] studied optimal control of a production-inventory system wih product returns.

\section{Preliminaries on Optimal Control}

Let $M$ be a smooth n-dimensional space, usually a subset of $R^{n}$. The main goal is to study a control system given by differential equations in the form

$$
\frac{d x^{i}}{d t}=f^{i}(x, u), \quad i=\overline{1, n},
$$

where $x=\left(x^{1}, \ldots, x^{n}\right) \in M$ represents the state of the system and $u \in U \subset R^{m}$ represents the controls $(m \leq n)$. A control system means a dynamical system evolving in state space and that can be controlled by the user. An optimal control problem consists of finding the trajectories of the control system which connects two states of the system $x_{0}$ and $x_{1}$ and minimizing the functional

$$
\min _{u} \int_{0}^{T} L(x(t), u(t)) d t, \quad x(0)=x_{0}, x(T)=x_{1},
$$

where $L$ is the Lagrangian or running cost (energy, cost, time, distance, etc.). The control theory deals with the study of systems whose evolution can be influenced by some external agents. The most important and powerful tool in the study of optimal solutions in control theory is provided by Pontryagin's Maximum Principle. It generates the differential equations of first order, which are only necessary for the optimal solutions. For each optimal trajectory, $c(t)=$ $(x(t), u(t))$ it offers a lift on the dual space $(x(t), p(t))$ satisfying Hamilton-Jacobi-Bellman equations. The Hamiltonian function has the form

$$
H(x, p, u)=\sum_{i=1}^{n} p_{i} f^{i}-L(x, u)
$$

where $\left(p_{1}, \ldots, p_{n}\right)$ are adjoint variables on dual space, while the maximization condition with respect to the control variables $u$, namely,

$$
H(x(t), p(t), u(t))=\max _{v} H(x(t), p(t), v(t))
$$

leads to $\frac{\partial H}{\partial u}=0$ ( $H$ is assumed to be smooth with respect to variable $u$ ). The extreme trajectories satisfy the equations

$$
\frac{d x^{i}}{d t}=\frac{\partial H}{\partial p_{i}}, \quad \frac{d p_{i}}{d t}=-\frac{\partial H}{d x^{i}} .
$$

Next, the case of control affine systems

(Brocket, [6]) given by

$$
\dot{x}=X_{0}+\sum_{i=1}^{m} u^{i} X_{i},
$$

is considered, where $\dot{x}=\frac{d x}{d t}$ and $X_{0}, X_{1}, \ldots, X_{m}$ are smooth vector fields on $M$. Usually, $X_{0}$ is called the drift vector field describing the dynamics of the system in the absence of controls and the vector fields $X_{1}, \ldots, X_{m}$ are called the input vector fields. The function $u(t)=\left(u^{1}(t), \ldots, u^{m}(t)\right)$ is the control or the input function, which may be specified freely in order to steer the system in a desired direction.

The system is controllable if for any two states $x_{0}$ and $x_{1}$ on $M$ there exists a finite time $T$ and an admissible control $u:[0, T] \rightarrow U$ so that for $x$ satisfying $x(0)=x_{0}$ results that $x(T)=x_{1}$. In other words, the system is controllable if for any two states $x_{0}$ and $x_{1}$ there exists a solution curve of (2) connecting $x_{0}$ and $x_{1}$. The controllability is the ability to steer a system from a given initial state to any final state, in finite time, using the available controls. Controllability does not care about the quality of the trajectory between two states, neither for the amount of control effort. In the following, a driftless control affine system, with $X_{0}=0$, is considered. In this case it results

$$
\dot{x}=\sum_{i=1}^{m} u^{i} X_{i}(x) .
$$

Most information about controllability is contained in the structure of the Lie algebra generated by the family of vector fields $X_{i}, i=\overline{1, m}$, which generate a distri- 
bution $\Delta=\operatorname{span}\left\{X_{1}, \ldots, X_{m}\right\}$. The distribution $\Delta$ has constant dimension $m$ if $\operatorname{dim} \Delta(x)=m$, for all points $x \in M$. It is known that the Lie bracket of two vector fields is given by $[X, Y](f)=X(Y(f))-Y(X(f))$.

The distribution is called involutive if the Lie bracket of any two vectors fields from $\Delta$ belongs to $\Delta$, that is $X_{i}, X_{j} \in \Delta \Rightarrow\left[X_{i}, X_{j}\right] \in \Delta$. It follows that in the case of involutive distribution, every Lie bracket of the vector fields can be expressed as a linear combination of the system vector fields, and therefore

$$
\left[X_{i}, X_{j}\right]=\sum_{k=1}^{m} L_{i j}^{k} X_{k}
$$

It is known that a foliation $\left\{S_{\alpha}\right\}_{\alpha \in I}$ of $M$ is a partition of $M=\bigcup_{\alpha \in I} S_{\alpha}$ of $M$ into disjoint connected subsets $S_{\alpha}$ called leaves. The well known Frobenius theorem says that if $\Delta$ is a distribution with constant dimension on $M$, then $\Delta$ is integrable if and only if $\Delta$ is involutive. In this case, the driftless control affine system is not controllable in the sense that the system can not be moved from an initial state to any final state. The distribution $\Delta$ determines a foliation on $M$ with the property that any curve is contained in a single leaf of the foliation. In other words, any two points can be joined by an optimal trajectory if and only if they are situated on the same leaf.

\section{Applications to Optimization and Production Planning}

It is supposed that a company can manufacture $n$ types of different products denoted $P_{1}, . ., P_{n}$. In a fixed period of time $T$, the company must produce a certain amount $\left(s_{1}, \ldots, s_{n}\right)$ of each type of products. It is known that some percentages from the quantities of the $P_{1}, . ., P_{n-1}$ products are used in the manufacture of the $P_{n}$ product, by a given law. It is assumed that the unit production costs decrease linearly with the production level (certain fixed costs are distributed across the entire quantity of products) and the cost of production operations for the last product $P_{n}$ is very small and can be neglected. In addition, the unit storage costs of holding inventory are given by $\left(\beta_{1}, \ldots, \beta_{n}\right)$ for each product. It is supposed that the production is not limited by lack of resources or production capacity. In these conditions, a plan of production is searched in order to ensure the required quantity at specified delivery data at minimum cost. The particular case of a single product is studied by Kamien and Schwartz [15].

Let $x^{i}=x^{i}(t), i=\overline{1, n}$ be the inventory accumulated by time $t$. By using the fact that the inventory level is the cumulated past production $p^{i}=p^{i}(t)$ and considering the initial stock null $x^{i}(0)=0$, the following is obtained:

$$
x^{i}(t)=\int_{0}^{t} p^{i}(s) d s .
$$

Hence, the rate of change of inventory level $\dot{x}^{i}$ is the production $p^{i}$ and we have $\dot{x}^{i}=p^{i}$. The unit production costs $c_{i}$ decrease linearly with the production level, and it results $c_{i}=\alpha_{i} p^{i}$, where $\alpha_{1}, \ldots, \alpha_{n-1} \in(0,1)$ are positive constants and the total cost of production is obtained

$$
\sum_{i=1}^{n-1} c_{i} p^{i}=\sum_{i=1}^{n-1} \alpha_{i}\left(p^{i}\right)^{2}=\sum_{i=1}^{n-1} \alpha_{i}\left(\dot{x}^{i}\right)^{2} .
$$

It results that the total cost, including the costs of holding inventory is given by

$$
\sum_{i=1}^{n-1} \alpha_{1}\left(\dot{x}^{i}\right)^{2}+\sum_{i=1}^{n-1} \beta_{i}\left(1-k_{i}\right) x^{i}+\beta_{n} x^{n},
$$

where $k_{1}, \ldots, k_{n-1} \in[0,1]$ represent the percentages from the quantities of $P_{1}, \ldots, P_{n-1}$ used in the manufacture of $P_{n}$. Next, considering $\dot{x}^{i}=u^{i}, i=\overline{1, n-1}$ the control variables and assuming that the rate of change of inventory for $P_{n}$ is given by the law

$$
\dot{x}^{n}=\sum_{i=1}^{n-1} k_{i} u^{i} x^{i}
$$

the following optimal control problem is obtained

$$
\left\{\begin{array}{l}
\dot{x}^{1}=u^{1} \\
\cdots \ldots \ldots \\
\dot{x}^{n-1}=u^{n-1} \\
\dot{x}^{n}=\sum_{i=1}^{n-1} k_{i} u^{i} x^{i} \\
x^{i}(0)=0 \\
x^{i}(T)=s_{i}, \quad i=\overline{1, n} \\
u^{1}, \ldots, u^{n-1} \in R \\
k_{1}, \ldots, k_{n-1} \in[0,1]
\end{array}\right.
$$


A plan of production with minimum cost

$$
\min _{u} \int_{0}^{T}\left(\sum_{i=1}^{n-1}\left(\alpha_{i}\left(u^{i}\right)^{2}+\beta_{i}\left(1-k_{i}\right) x^{i}\right)+\beta_{n} x^{n}\right) d t .
$$

is the solution. It results that this is a driftless control affine system on $M=R_{+}^{n}$, which can be written in the form

$$
\begin{aligned}
& \dot{x}=\sum_{i=1}^{n-1} u^{i} X_{i}, \quad x=\left(x^{1}, \ldots, x^{n}\right)^{t} \in R_{+}^{n} \\
& \min _{u} \int_{0}^{T} F(u(t), x(t)) d t,
\end{aligned}
$$

where

$$
\begin{aligned}
& F(u(t), x(t))=\sum_{i=1}^{n-1}\left(\alpha_{i}\left(u^{i}\right)^{2}+\beta_{i}\left(1-k_{i}\right) x^{i}\right)+\beta_{n} x^{n} . \\
& X_{1}=\left(\begin{array}{c}
1 \\
0 \\
\vdots \\
k_{1} x^{1}
\end{array}\right), X_{2}=\left(\begin{array}{c}
0 \\
1 \\
\vdots \\
k_{2} x^{2}
\end{array}\right), \ldots, X_{n-1}=\left(\begin{array}{c}
0 \\
\vdots \\
1 \\
k_{n-1} x^{n-1}
\end{array}\right) .
\end{aligned}
$$

The optimal solutions starting from the initial point $(0, \ldots, 0)$ to endpoint $\left(s_{1}, \ldots, s_{n}\right)$ are sought. The distribution $\Delta=\operatorname{span}\left\{X_{1}, \ldots, X_{n-1}\right\}$ generated by the vector fields $X_{1}, \ldots, X_{n-1}$ has constant dimension, $\operatorname{dim} \Delta(x)=n-1$, for all $x \in R^{n}$. In the natural basis $\left\{\frac{\partial}{\partial x^{1}}, \ldots, \frac{\partial}{\partial x^{n}}\right\}$ of $R^{n}$, the vector fields have the expressions

$$
\begin{aligned}
& X_{1}=\frac{\partial}{\partial x^{1}}+k_{1} x^{1} \frac{\partial}{\partial x^{n}}, \ldots \\
& X_{n-1}=\frac{\partial}{\partial x^{n-1}}+k_{n-1} x^{n-1} \frac{\partial}{\partial x^{n}}
\end{aligned}
$$

By using the following Lie bracket formula

$$
[f X, g Y]=f g[X, Y]+f X(g) Y-g Y(f) X,
$$

every Lie bracket of two vectors from distribution is given by

$$
\left[X_{i}, X_{j}\right]=\left[\frac{\partial}{\partial x^{i}}+k_{i} x^{i} \frac{\partial}{\partial x^{n}}, \frac{\partial}{\partial x^{j}}+k_{j} x^{j} \frac{\partial}{\partial x^{n}}\right]=0,
$$

and it results that the distribution $\Delta$ is involutive. Using the Frobenius theorem, it results that the distribution is integrable and as a consequence, it determines a foliation on state space $R_{+}^{n}$. Consequently, two points can be joined by an optimal trajectory if and only if they are situated on the same leaf. It results that the economical system is not controllable, in the sense that it is not possible to manufacture any quantity required. Indeed, by using Equation (4), the following is obtained

$$
\dot{x}^{n}=\sum_{i=1}^{n-1} k_{i} x^{i} \dot{x}^{i},
$$

and through integration, it results

$$
x^{n}=\frac{1}{2} \sum_{i=1}^{n-1} k_{i}\left(x^{i}\right)^{2}+c, \quad c \in R,
$$

which are the hipersurfaces in $R_{+}^{n}$, which determine a foliation. In the three-dimensional case, the foliation is generated by surfaces which are elliptic paraboloids.

Moreover, by using that $x^{i}(0)=0$, one gets the relation

$$
x^{n}=\frac{1}{2} \sum_{i=1}^{n-1} k_{i}\left(x^{i}\right)^{2},
$$

and from $x^{i}(T)=s_{i}$ results that the problem has a solution (the system is controllable), so it is possible to manufacture the quantity $\left(s_{1}, s_{2}, \ldots, s_{n}\right)$ if and only if the final amounts satisfy the condition

$$
s_{n}=\frac{1}{2} \sum_{i=1}^{n-1} k_{i}\left(s_{i}\right)^{2} .
$$

In other words, the system is not controllable if the previous condition is not satisfied.

The main result of the paper is the following:

Theorem. The optimal solution of the control system is given by

$$
\begin{aligned}
& x^{i}(t)=c_{i_{1}} e^{\sqrt{\frac{k_{i} \beta_{n}}{2 \alpha_{i}}} t}+c_{i_{2}} e^{-\sqrt{\frac{k_{i} \beta_{n}}{2 \alpha_{i}}} t}-\frac{\beta_{i}\left(1-k_{i}\right)}{k_{i} \beta_{n}}, i=\overline{1, n-1}, \\
& x^{n}=\frac{1}{2} \sum_{i=1}^{n-1} k_{i}\left(x^{i}\right)^{2},
\end{aligned}
$$


where

$$
\begin{aligned}
& c_{i_{1}}=\frac{1-k_{i}}{d_{i}+1} \frac{\beta_{i}}{k_{i} \beta_{n}}+\frac{d_{i} s_{i}}{d_{i}^{2}-1}, \\
& c_{i_{2}}=\frac{d_{i}}{d_{i}+1} \frac{\beta_{i}\left(1-k_{i}\right)}{k_{i} \beta_{n}}-\frac{d_{i} s_{i}}{d_{i}^{2}-1}, \\
& d_{i}=e^{\sqrt{\frac{k_{i} \beta_{n}}{2 \alpha_{i}}} T},
\end{aligned}
$$

and optimal control variables have the form

$$
u^{i}(t)=\sqrt{\frac{k_{i} \beta_{n}}{2 \alpha_{i}}}\left(c_{i_{1}} e^{\sqrt{\frac{k_{i} \beta_{n}}{2 \alpha_{i}}} t}-c_{i_{2}} e^{-\sqrt{\frac{k_{i} \beta_{n}}{2 \alpha_{i}}} t}\right) .
$$

Proof. The Pontryagin Maximum Principle will be used in order to find the optimal solution. The Hamiltonian function on dual space is given by

$$
\begin{aligned}
& H=\sum_{i=1}^{n} p_{i} \dot{x}^{i}-F \text { which leads to } \\
& H=\sum_{i=1}^{n-1} p_{i} u^{i}+p_{n} \sum_{i=1}^{n-1} k_{i} u^{i} x^{i} \\
& -\sum_{i=1}^{n-1}\left(\alpha_{i}\left(u^{i}\right)^{2}+\beta_{i}\left(1-k_{i}\right) x^{i}\right)-\beta_{n} x^{n} .
\end{aligned}
$$

The condition $\frac{\partial H}{\partial u^{i}}=0, i=\overline{1, n-1}$ yields the follow-
ing equations

$$
p_{i}+p_{n} k_{i} x^{i}-2 \alpha_{i} u^{i}=0 \Rightarrow u^{i}=\frac{p_{i}+p_{n} k_{i} x^{i}}{2 \alpha_{i}} .
$$

Now, replacing the expressions of the control variables $u^{1}, \ldots, u^{n-1}$ into the expression of the Hamiltonian, by straightforward computation it results

$$
H=\sum_{i=1}^{n-1}\left(\frac{\left(p_{i}+p_{n} k_{i} x^{i}\right)^{2}}{4 \alpha_{1}}-\beta_{i}\left(1-k_{i}\right) x^{i}\right)-\beta_{n} x^{n} .
$$

Using the Hamilton-Jacobi-Bellman Equations (1) the following system of first order differential equations is obtained:

$$
\begin{aligned}
& \dot{x}^{i}=\frac{\partial H}{\partial p_{i}}=\frac{p_{i}+p_{n} k_{i} x^{i}}{2 \alpha_{i}}, \quad i=\overline{1, n-1} \\
& \dot{x}^{n}=\frac{\partial H}{\partial p_{n}}=\sum_{i=1}^{n-1} \frac{\left(p_{i}+p_{n} k_{i} x^{i}\right) k_{i} x^{i}}{2 \alpha_{i}} \\
& \dot{p}_{i}=-\frac{\partial H}{\partial x^{i}}=\beta_{i}\left(1-k_{i}\right)-\frac{\left(p_{i}+p_{n} k_{i} x^{i}\right) p_{n} k_{i}}{2 \alpha_{i}}, \\
& \dot{p}_{n}=-\frac{\partial H}{\partial x^{n}}=\beta_{n} .
\end{aligned}
$$

In order to solve this system of differential equations the following change of variables is considered

$$
\mu_{i}=p_{i}+p_{n} k_{i} x^{i}, \quad i=\overline{1, n-1}
$$

which leads to

$$
\begin{aligned}
\dot{x}^{i} & =\frac{\mu_{i}}{2 \alpha_{i}}, \quad i=\overline{1, n-1}, \\
\dot{x}^{n} & =\sum_{i=1}^{n-1} \frac{\mu_{i} k_{i} x^{i}}{2 \alpha_{i}} .
\end{aligned}
$$

Moreover, by direct computation the following can be obtain

$$
\begin{aligned}
& \dot{\mu}_{i}=\dot{p}_{i}+\dot{p}_{n} k_{i} x^{i}+p_{n} k_{i} \dot{x}^{i} \\
& =\beta_{i}\left(1-k_{i}\right)-\frac{\left(p_{i}+p_{n} k_{i} x^{i}\right) p_{n} k_{i}}{2 \alpha_{i}}+\beta_{n} k_{i} x^{i} \\
& +\frac{\left(p_{i}+p_{n} k_{i} x^{i}\right) p_{n} k_{i}}{2 \alpha_{i}}=\beta_{i}\left(1-k_{i}\right)+\beta_{n} k_{i} x^{i} .
\end{aligned}
$$

In addition, from (6), it results

$$
\ddot{x}^{i}=\frac{\dot{\mu}_{i}}{2 \alpha_{i}}=\frac{\beta_{n} k_{i} x^{i}}{2 \alpha_{i}}+\frac{\beta_{i}\left(1-k_{i}\right)}{2 \alpha_{i}},
$$

that is a linear nonhomogeneous second order differential equation. Considering the corresponding linear homogeneous second order differential equation

$$
\ddot{x}^{i}-\frac{k_{i} \beta_{n}}{2 \alpha_{i}} x^{i}=0,
$$


and using the characteristic equation $\lambda^{2}-\frac{k_{i} \beta_{n}}{2 \alpha_{i}}=0$, with solutions $\lambda_{1,2}= \pm \sqrt{\frac{k_{i} \beta_{n}}{2 \alpha_{i}}}$, the general solution of the homogeneous second order differential equation is obtained

$$
x^{i}(t)=c_{i_{1}} e^{\sqrt{\frac{k_{i} \beta_{n}}{2 \alpha_{i}}} t}+c_{i_{2}} e^{-\sqrt{\frac{k_{i} \beta_{n}}{2 \alpha_{i}}} t} .
$$

Hence, the general solution of the nonhomogeneous second order differential equation is given by

$$
x^{i}(t)=c_{i_{1}} e^{\sqrt{\frac{k_{i} \beta_{n}}{2 \alpha_{i}}} t}+c_{i_{2}} e^{-\sqrt{\frac{k_{i} \beta_{n}}{2 \alpha_{i}}} t}-\frac{\beta_{i}\left(1-k_{i}\right)}{k_{i} \beta_{n}},
$$

$i=\overline{1, n-1}, \quad x^{n}=\frac{1}{2} \sum_{i=1}^{n-1} k_{i}\left(x^{i}\right)^{2}$.

This solution of control system is optimal because the Hamiltonian function is convex. Using the initial conditions $x^{i}(0)=0, x^{i}(T)=s_{i}, \quad i=\overline{1, n}$ the linear system

$$
\left\{\begin{array}{c}
c_{i_{1}}+c_{i_{2}}=\frac{\beta_{i}\left(1-k_{i}\right)}{k_{i} \beta_{n}} \\
c_{i_{1}} d_{i}+\frac{c_{i_{2}}}{d_{i}}=\frac{\beta_{i}\left(1-k_{i}\right)}{k_{i} \beta_{n}}+s_{i},
\end{array}\right.
$$

is obtained, where $d_{i}=e^{\sqrt{\sqrt{k_{i} \beta_{n}}} T}>1$. The solution of the system (8) is given by

$$
\begin{aligned}
c_{i_{1}} & =\frac{1-k_{i}}{d_{i}+1} \frac{\beta_{i}}{k_{i} \beta_{n}}+\frac{d_{i} s_{i}}{d_{i}^{2}-1}, \\
c_{i_{2}} & =\frac{d_{i}}{d_{i}+1} \frac{\beta_{i}\left(1-k_{i}\right)}{k_{i} \beta_{n}}-\frac{d_{i} s_{i}}{d_{i}^{2}-1} .
\end{aligned}
$$

Next, by introducing the expression of $x^{i}$ from ( 7 ), into equations $\dot{\mu}_{i}=\beta_{i}\left(1-k_{i}\right)+\beta_{n} k_{i} x^{i}$ it results

$$
\dot{\mu}_{i}(t)=k_{i} \beta_{n} c_{i_{1}} e^{\sqrt{\frac{k_{i} \beta_{n}}{2 \alpha_{i}}} t}+k_{i} \beta_{n} c_{i_{2}} e^{-\sqrt{\frac{k_{i} \beta_{n}}{2 \alpha_{i}}} t},
$$

which yields $\mu_{i}(t)=\sqrt{2 \alpha_{i} k_{i} \beta_{n}}\left(c_{i_{1}} e^{\sqrt{\frac{k_{i} \beta_{n}}{2 \alpha_{i}}} t}-c_{i_{2}} e^{-\sqrt{\frac{k_{i} \beta_{n}}{2 \alpha_{i}}} t}\right)+a_{i}$.

Now, one can find the control variables, for every $i=\overline{1, n-1}$.

$$
u^{i}=\frac{\mu_{i}}{2 \alpha_{i}}=\sqrt{\frac{k_{i} \beta_{n}}{2 \alpha_{i}}}\left(c_{i_{1}} e^{\sqrt{\frac{k_{i} \beta_{n}}{2 \alpha_{i}}} t}-c_{i_{2}} e^{-\sqrt{\frac{k_{i} \beta_{n}}{2 \alpha_{i}}} t}\right)+\frac{a_{i}}{2 \alpha_{1}} .
$$

Moreover, by using equation

$$
x^{i}(T)-x^{i}(0)=\int_{0}^{T} \dot{x}^{i}(t) d t=\int_{0}^{T} u^{i}(t) d t,
$$

the following can be obtained:

$$
s_{i}=\int_{0}^{T}\left(\sqrt{\frac{k_{i} \beta_{n}}{2 \alpha_{i}}}\left(c_{i_{1}} e^{\sqrt{\frac{k_{i} \beta_{n}}{2 \alpha_{i}}} t}-c_{i_{2}} e^{-\sqrt{\frac{k_{i} \beta_{n}}{2 \alpha_{i}}} t}\right)+\frac{a_{i}}{2 \alpha_{i}}\right) d t \text {, }
$$

and it results

$$
S_{i}=c_{i_{1}}\left(e^{\sqrt{\frac{k_{i} \beta_{n}}{2 \alpha_{i}}} T}-1\right)+c_{i_{2}}\left(e^{-\sqrt{\frac{k_{i} \beta_{n}}{2 \alpha_{i}}} T}-1\right)+\frac{a_{i}}{2 \alpha_{i}} T,
$$

which yields

$$
a_{i}=\frac{2 \alpha_{i}}{T}\left(s_{i}-c_{i_{i}}\left(e^{\sqrt{\frac{k_{i} \beta_{n}}{2 \alpha_{i}}} T}-1\right)-c_{i_{2}}\left(e^{-\sqrt{\frac{k_{i} \beta_{n}}{2 \alpha_{i}}} T}-1\right)\right) \text {, }
$$

However, from (8) it results

$$
\begin{aligned}
& a_{i}=\frac{2 \alpha_{i}}{T}\left(s_{i}-c_{i_{i}}\left(d_{i}-1\right)-c_{i_{2}}\left(\frac{1}{d_{i}}-1\right)\right) \\
& =\frac{2 \alpha_{i}}{T}\left(-\frac{\beta_{i}\left(1-k_{i}\right)}{k_{i} \beta_{n}}+\frac{\beta_{i}\left(1-k_{i}\right)}{k_{i} \beta_{n}}\right)=0 .
\end{aligned}
$$

and finally, the control variables are obtained, for $i=\overline{1, n-1}$

$$
u^{i}(t)=\sqrt{\frac{k_{i} \beta_{n}}{2 \alpha_{i}}}\left(c_{i_{1}} e^{\sqrt{\frac{k_{i} \beta_{n}}{2 \alpha_{i}}} t}-c_{i_{2}} e^{-\sqrt{\frac{k_{i} \beta_{n}}{2 \alpha_{i}}} t}\right),
$$

which ends the proof. 


\section{Numerical Examples}

\section{First example:}

The following numerical example in three dimensional case $n=3$ is given by:

- fixed period of time is $T=1$,

- final stock quantities from products $P_{1}, P_{2}, P_{3}$ are given by $s_{1}=4, s_{2}=6, s_{3}=13$,

- storage costs are $\beta_{1}=4, \beta_{2}=3, \beta_{3}=2$ and the coefficients $\alpha_{1}=0.5, \alpha_{2}=0.5, k_{1}=0.5, k_{2}=0.5$.

This economical system is controllable, because the final quantities satisfy the condition

$$
s_{3}=\frac{k_{1} s_{1}^{2}+k_{2} s_{2}^{2}}{2} .
$$

The optimal solutions of control system are given by the relations (7) and are shown in Figures 1-4. The optimal control variables are given by the relations (9) and are represented in Figures 5-6.

It should be noted that the optimal solutions satisfy the initial stock conditions

$x^{1}(0)=0, x^{2}(0)=0, x^{3}(0)=0$, and final stock conditions $x^{1}(1)=4, x^{2}(1)=6, x^{3}(1)=13$

The quantities of products $P_{1}, P_{2}, P_{3}$ increase in time as shown in Figures 1-4.

Moreover, the production rate for the second product is higher than for the first product, a fact which also results from the Figures 5-6.

\section{Second example:}

The following numerical example for the case of four products $n=4$ is given by:

- fixed period of time is $T=2$,

- final stock quantities from products $P_{1}, P_{2}, P_{3}, P_{4}$ are given by $s_{1}=10, s_{2}=12, s_{3}=15, s_{4}=70$,

- storage costs are $\beta_{1}=2, \beta_{2}=1, \beta_{3}=2, \beta_{4}=3$, and the coefficients $\alpha_{1}=0.1, \alpha_{2}=0.2, \alpha_{2}=0.5, k_{1}=0.1, k_{2}=0.5$, $k_{3}=0.2$.

This system is not controllable, because

$$
s_{4} \neq \frac{k_{1} s_{1}^{2}+k_{2} s_{2}^{2}+k_{3} s_{3}^{2}}{2}=63.5
$$

The final stock for the products $P_{1}, P_{2}, P_{3}$ given by $s_{1}=$ $10, s_{2}=12, s_{3}=15$ can be obtained, with solution from (7), but the final stock for $P_{4}$ can not be touched. The system is controllable if $s_{4}=63.5$.

\section{Figure 1}

Optimal solution $x^{1}(t), t \in[0,1]$

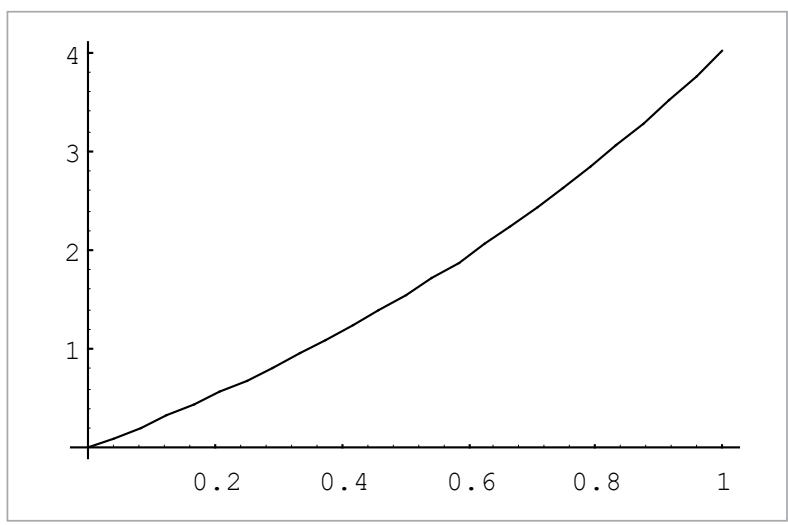

Figure 2

Optimal solution $x^{2}(t), t \in[0,1]$

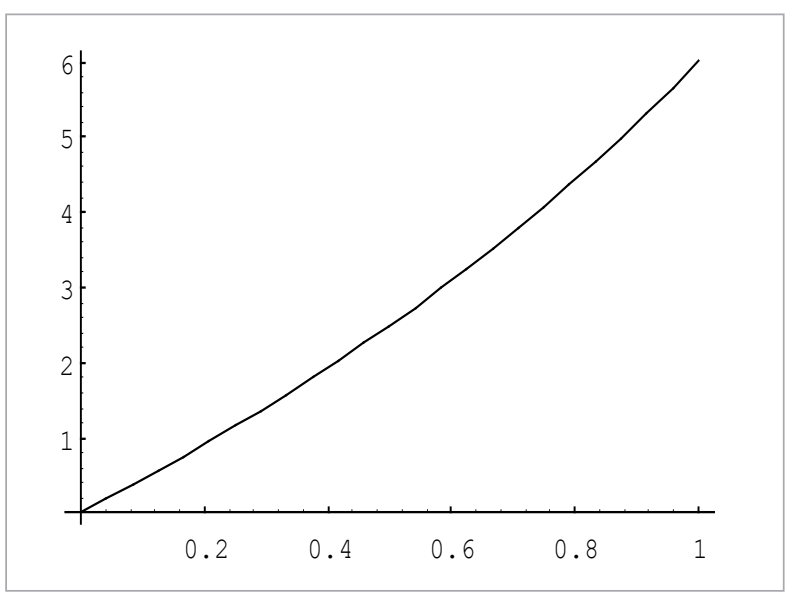

Figure 3

Optimal solution $x^{3}(t), t \in[0,1]$

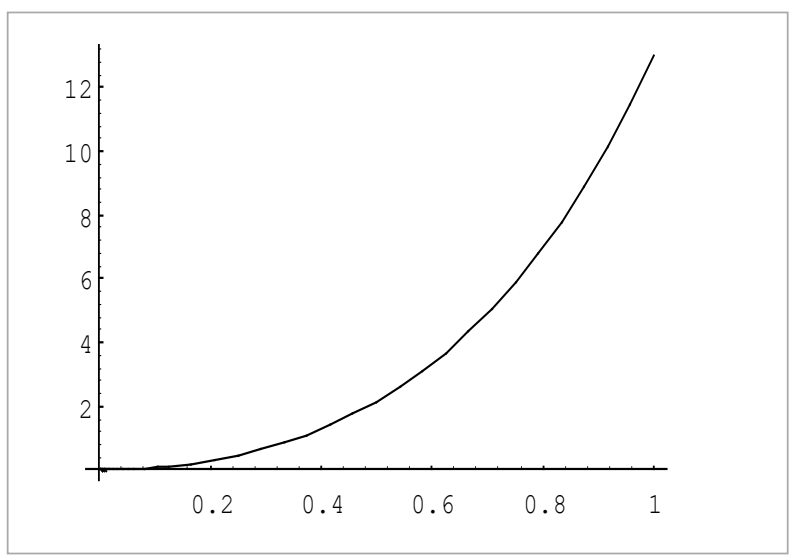


Figure 4

Optimal solutions $\left(x^{1}(t), x^{2}(t), x^{3}(t)\right) t \in[0,1]$

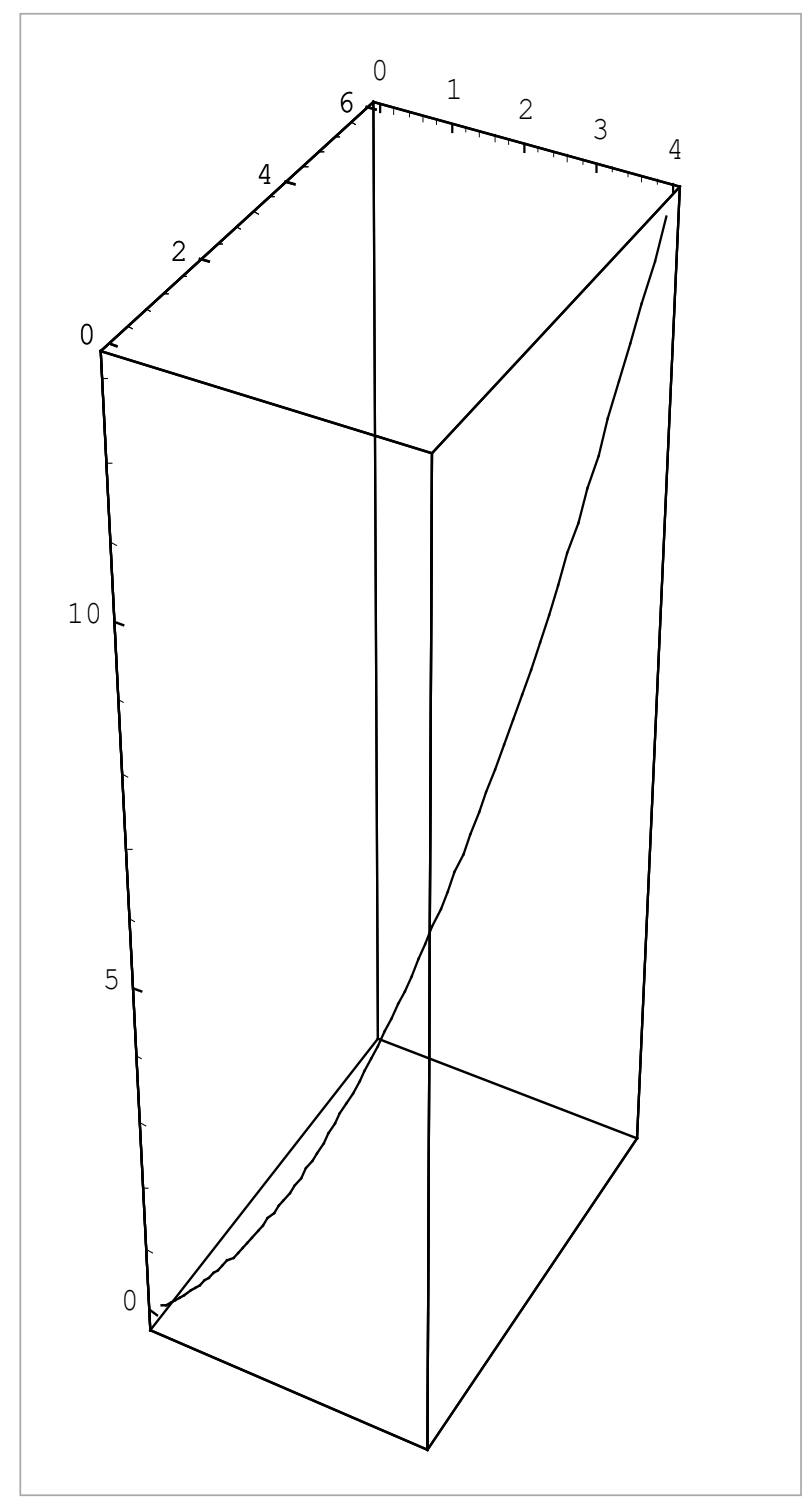

Figure 5

Optimal control variable $u^{1}(t), t \in[0,1]$

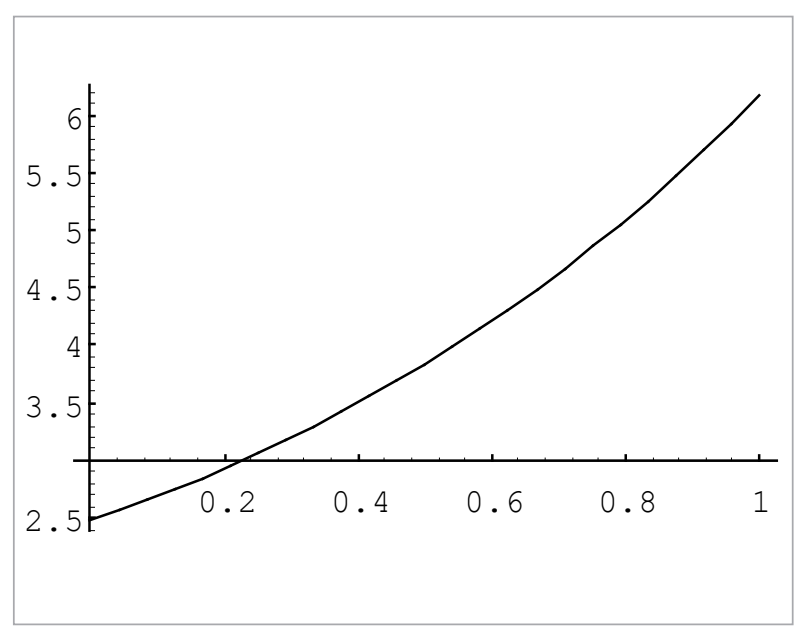

Figure 6

Optimal control variable $u^{2}(t), t \in[0,1]$

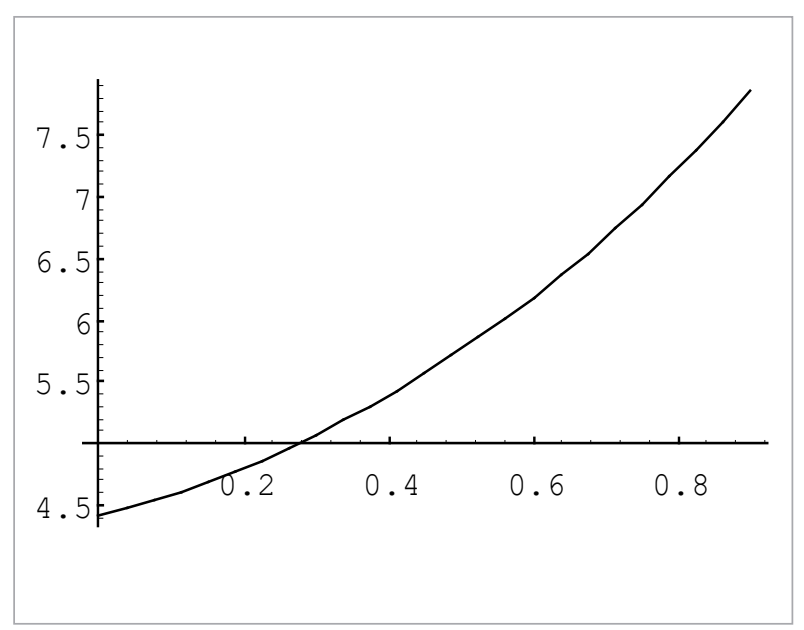

\section{Conclusions}

In this paper a mathematical model for an economic problem is proposed, that consists in manufacturing a number of $n$ products at a specified date so that the production and storage costs are minimal. The paper incorporates the problem into an optimal control model and applies the Pontryagin Maximum Princi-

ple to find the optimal solutions. In order to solve the system of differential equations, a convenient change of variables is considered. It is interesting to see that the economic system is not controllable, in the sense that it is not possible to reach any final stock quantity. Finally, two numerical examples are given. 


\section{References}

1. Agrachev, A., Sachkov, Y. L. Control Theory from the Geometric Viewpoint. Encyclopedia of Mathematical Sciences, Control Theory and Optimization, 87, Berlin, Springer-Verlag, 2004. https://doi.org/10.1007/978-3662-06404-7

2. Arrow, K. J. Applications of Control Theory of Economic Growth. Mathematics of Decision Sciences, 2, AMS, 1968.

3. Axsäter, S. Control Theory Concepts in Production and Inventory Control. International Journal of Systems Science, 1985, 16(2), 161-169. https://doi. org/10.1080/00207728508926662

4. Bellmann, R. Adaptive Control Processes: a guided tour. Princeton Univ. Press: Princeton, 1972.

5. Benjaafar, S., Gayon, J. P., Tepe, S. Optimal Control of a Production-Inventory System with Customer Impatience. Operations Research Letters, 2010, 38(4), 267272. https://doi.org/10.1016/j.orl.2010.03.008

6. Brocket, R. Lie Algebra and Lie Groups in Control Theory. Geometrical Methods in System Theory, Dordrecht, 1973, 43-82. https://doi.org/10.1007/978-94-010-26758_2

7. Caputo, M. Foundations of Dynamic Economic Analysis: Optimal Control Theory and Applications, Cambridge University Press, 2005. https://doi.org/10.1017/ CBO9780511806827

8. Dolgui A., Ben Ammar, O., Hnaein, F., Louly, M. State of the Art on Supply Planning and Inventory Control under Lead Time Uncertainty. Studies in Informatics and Control, (2013), 22(3), 255-268. https://doi. org/10.24846/v22i3y201302

9. Feichtinger, G., Hartl, R. Optimal Pricing and Production in an Inventory Model. European Journal of Operational Research, 1985, 19, 45-56. https://doi. org/10.1016/0377-2217(85)90307-8

10. Gaimon, C. Simultaneous and Dynamic Price, Production, Inventory and Capacity Decisions. European Journal of Operational Research, 1988, 35, 426-441. https://doi.org/10.1016/0377-2217(88)90232-9

11. Gayon, J. P., Vercraene, S., Flapper, S. D. Optimal Control of a Production-Inventory System with Product Returns and Two Disposal Options. European Journal of Operational Research, 2017, 262(2), 499-508. https:// doi.org/10.1016/j.ejor.2017.03.018

12. Hermosilla, C. Vinter, R. Zidani, H., Hamilton-Jacobi-Bellman Equations for Optimal Control Processes with Convex State Constraints. Systems \& Control Letters, 2017, 109, 30-36. https://doi.org/10.1016/j.sysconle.2017.09.004

13. Hwang, C. L., Fan, L. T., Erikson, L. E. Optimum Production Planning by the Maximum Principle. Management Science, 1967, 13(9), 751-755. https://doi.org/10.1287/ mnsc.13.9.751

14. Janušauskaitè, N. The Mathematical Models for the Multistage Inventory Control Processes. Information Technology and Control, 2004, 33(4), 77-81. https://doi. org/10.5755/j01.itc.33.4.11950

15. Kamien, M. I., Schwartz, N. L. Dynamic Optimization. Elsevier, 2006.

16. Kogan, K., Khmelnitsky, E. An Optimal Control Model for Continuous Time Production and Setup Scheduling. International Journal of Production Research, 1996, 34(3), 715 -725. https://doi. org/10.1080/00207549608904930

17. LaValle, S. M. Planning Algorithms. Cambridge University Press, 2006. https://doi.org/10.1017/ CBO9780511546877

18. Lee, E. B., Markus, L. Foundations of Optimal Control Theory. John Wiley \& Sons, New York, 1967.

19. Milosz, M., Murzabekov, Z., Tussupova, K., Usubalieva, S. Optimisation of Discrete Processes with Bounded Control. Information Technology and Control, 2018, 47(4), 684-690. https://doi.org/10.5755/j01. itc. 47.4 .19933

20. Ortega, M., Lin, L. Control Theory Applications to the Production-Inventory Problem: A Review. International Journal of Production Research, 2004, 42(11), 23032322. https://doi.org/10.1080/00207540410001666260

21. Pontryagin, L. S., Boltyanskii, V. G., Gamkrelidze, R. V., Mishchenko, E. F. The Mathematical Theory of Optimal Processes, John Wiley \& Sons, 1962.

22. Popescu, L. Applications of Driftless Control Affine Systems to a Problem of Inventory and Production. Studies in Informatics and Control, 2019, 28(1), 25-34. https:// doi.org/10.24846/v28i1y201903

23. Seierstad, A., Sydsater, K. Optimal Control Theory with Economic Applications. North-Holland, Amsterdam, $198 \%$.

24. Sethi, S. P. A Survey of Management Science Applications of the Deterministic Maximum Principle. Studies in the Management Science, 1978, 9, 33-68. 
25. Sethi, S. P. Applications of the Maximum Principle to Production and Inventory Problems. Proceedings Third International Symposium on Inventories, Budapest, 1984, 753-756.

26. Sethi, S. P., Thompson, G. L. Optimal Control Theory: Applications to Management Science and Economics. Springer, New York, 2000.

27. Sontag, E. Mathematical Control Theory: Deterministic Finite Dimensional Systems. Springer, 1998.

28. Schwartz, J. D., Rivera, D. E. A Process Control Approach to Tactical Inventory Management in Produc-
tion-Inventory Systems. International Journal of Production Economics, 2010, 125(1), 111-124. https://doi. org/10.1016/j.ijpe.2010.01.011

29. Varjani, M. M, Shamsi M., Malek, A. Solving a Class of Hamilton-Jacobi-Belmann Equations Using Pseudospectral Methods. Kybernetica, 2018, 54, 629-64\%. https://doi.org/10.14736/kyb-2018-4-0629

30. Weber, T. A. Optimal Control Theory with Applications in Economics. MIT Press, 2011. https://doi.org/10.7551/ mitpress/9780262015738.001.0001 